\title{
Age and Helicobacter pylori decrease gastric mucosal surface hydrophobicity independently
}

\author{
A Hackelsberger, U Platzer, M Nilius, V Schultze, T Günther, J E Dominguez-Muñoz, \\ $P$ Malfertheiner
}

\begin{abstract}
Background-Gastric mucosal surface hydrophobicity (GMSH) is an essential component of the mucosal defence system that is decreased by Helicobacter pylori and non-steroidal anti-inflammatory drugs (NSAIDs). Gastric ulcers occur predominantly in elderly subjects, and may thus reflect diminished mucosal resistance.

Aims-To investigate whether aging decreases GMSH.

Patients-One hundred and twenty patients without peptic ulcer disease were divided into three age groups: I (41 years or below); II (41-64 years); and III (65 years or above).

Methods-Biopsy specimens were taken from the antrum, corpus, and cardia for histology (Sydney system), urease testing for $H$ pylori, and for contact angle measurement of GMSH with a goniometer. The presence of specific $H$ pylori antibodies was checked by immunoblotting.

Results-Fifty two patients (43\%) were infected, and 68 were uninfected with $H$ pylori. GMSH at all biopsy sites was lower in $H$ pylori infected subjects $(p=0.0001)$, but also decreased with age independently of infection status $(p=0.0001)$. The most notable decrease in GMSH occurred between age groups I and II in those with, and between age groups II and III in those without, $H$ pylori infection. GMSH was greater in antral than in corpus mucosa in both infected $(p=0.0001)$ and uninfected patients $(p=0.0003)$.

Conclusions-A physiological decrease in GMSH with aging may contribute to the risk of ulcer development in the elderly, and may act synergistically with $H$ pylori and/or NSAIDs on gastric mucosal defence.

(Gut 1998;43:465-469)
\end{abstract}

Keywords: gastric mucosal defence; surface hydrophobicity; aging; Helicobacter pylori

The pathogenesis of peptic ulcer disease (PUD) is considered to be an imbalance of defensive and aggressive factors in the upper gastrointestinal tract. Helicobacter pylori is a key pathogenetic factor in peptic ulceration ${ }^{1}$ as are non-steroidal anti-inflammatory drugs (NSAIDs) $^{2}$; each factor acts independently of the other. A substantial proportion of gastric ulcer patients may, however, both have $H$ pylori gastritis and use NSAIDs. ${ }^{3}$ While gastric acid output may be modified by $H$ pylori gastritis towards either hypersecretion or hyposecretion, ${ }^{45}$ it is not altered by NSAIDs. Like $H$ pylori gastritis the latter compromise the "defence line" of the gastric mucosa. The specific hydrophobic property of the gastric mucosa is an essential component of the mucosal barrier and is due to an adsorbed layer of surface active phospholipids (SAPL) ${ }^{6}$ secreted by gastric mucous cells. ${ }^{7}$ Gastric mucosal surface hydrophobicity (GMSH) can be measured reliably and quite simply as the contact angle at which a drop of saline intersects the surface of the gastric mucosa. ${ }^{8}$ This technique has been applied to endoscopic biopsy specimens of gastric mucosa, ${ }^{9}$ and it has been shown that both $H$ pylori gastritis ${ }^{10}$ and NSAIDs $^{11}$ decrease GMSH. While $H$ pylori infection is acquired in childhood in most individuals so affected, ${ }^{12}{ }^{13}$ gastric ulcer is a disease of the middle aged and the elderly. ${ }^{14-16}$ In patients on NSAID medication the risk of gastric ulcer complications is also clearly increased in the elderly. ${ }^{17} 18$ This investigation therefore addressed the question whether, apart from $H$ pylori infection, aging itself decreases GMSH, and whether differences in GMSH occur in antral, corpus, and cardiac mucosa.

\section{Materials and methods}

PATIENT SELECTION

During the eight month period between October 1995 and May 1996, 135 patients undergoing elective upper gastrointestinal endoscopy at our department were recruited. All underwent a standardised interview to check inclusion criteria, and gave their informed consent to the extended biopsy protocol, which was approved by the local hospital ethics committee. Patients with a history of PUD, gastric surgery, malignancy, coagulation disorders, or medication with NSAIDs, corticosteroids, antibiotics, bismuth, histamine $\mathrm{H}_{2}$ receptor antagonists, or proton pump inhibitors (PPI) during the month preceding endoscopy were excluded, as were those previously examined for $H$ pylori status. We also excluded all smokers, regular drinkers, and patients with severe diseases outside the upper gastrointestinal tract. Four months into the study a provisional analysis of the age distribution of the patients participating showed a preponderance of middle aged subjects; from then only younger or older patients were admitted.

\section{ENDOSCOPY}

Endoscopy was performed after an overnight fast. If no significant lesions (ulcers, malignant
Accepted for publication 23 March 1998 
disease, varices, Barrett's oesophagus) were found (up to three chronic erosions were accepted) patients were included. Closely grouped mucosal biopsy specimens were obtained from each gastric compartment with standard forceps: four specimens from the antrum ( $2 \mathrm{~cm}$ from the pylorus), four from the corpus (mid region of the greater curvature), and three from the cardia directly below the squamocolumnar junction. After endoscopy a blood sample was obtained.

GASTRIC MUCOSAL SURFACE HYDROPHOBICITY The measurement was performed as previously described and validated. ${ }^{9}$ In brief, an unfixed biopsy specimen from each site was rinsed with $0.9 \%$ saline, oriented mucosa upwards on a glass slide under a dissecting microscope, and placed on the specimen stage of the goniometer (Rame-Hart 100-00, Mountain Lakes, New York). After 30 minutes air drying, a $0.5 \mu \mathrm{l}$ drop of $0.9 \%$ saline was applied to the mucosal surface and the plateau contact angle at the liquid/solid interface as an index of GMSH was read on the left and right side of the droplet. Three droplets were thus applied to give six measurements on each specimen, and the mean value was calculated. A measurement was judged to be unreliable if variations of more than $5^{\circ}$ were found on a biopsy specimen, and the patient was then excluded. The investigator performing the measurements (UP) was unaware of the patient's age.

\section{HISTOLOGY}

After fixation in $4 \%$ neutral formalin, $4 \mu \mathrm{m}$ sections from two biopsy specimens obtained from each gastric compartment were prepared by routine methods and stained with modified Giemsa to detect $H$ pylori and grade the bacterial density, and with haematoxylin and eosin to grade the the degree and activity of gastritis. A four point scale (0-3) was used for all gradings in accordance with the updated Sydney system. ${ }^{19}$

\section{ASSESSMENT OF H PYLORI INFECTION}

We additionally used a previously validated ${ }^{20}$ rapid urease test (HUT-test, Astra $\mathrm{GmbH}$, Wedel, Germany) for the enzymatic detection of $H$ pylori in one antral and one corpus biopsy specimen. We also used a previously validated ${ }^{21}$ commercially available western blot test (Helico-Blot 2.0, Genelabs, Singapore) to detect antibodies to CagA, VacA, and four further specific $H$ pylori proteins in all patients. $H$ pylori infection was assessed if active gastritis with bacteria of typical shape was shown by histology and/or the rapid urease test read positive. Patients in whom serological but no biopsy based evidence of $H$ pylori infection was found were excluded from the evaluation.

STATISTICAL ANALYSIS

Variables associated with GMSH were assessed by analysis of variance (ANOVA), $t$ test, and $\mathrm{U}$ test and Spearman's correlations using the SPSS package. Significance was considered at a $5 \%$ probability level.

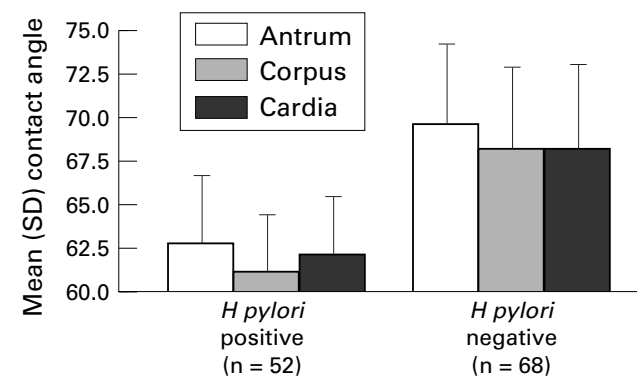

Figure 1 Means of contact angles as a function of $H$ pylori status and biopsy site.

\section{Results}

In eight of 135 patients enrolled the contact angle measurements were considered unreliable on the criteria described earlier. In most of these cases the biopsy specimens had an irregular surface and the patients were excluded. A further seven patients with uncertain $H$ pylori status were also excluded. A total of 120 patients (65 females (54\%)) with a mean age of 52.2 (19.7) years (range 16-85) were finally evaluated. Fifty two patients $(43 \%)$ were infected with $H$ pylori and were significantly older (mean age $59.4(17.5)$ years, $\mathrm{p}<0.0001$ ) than the 68 uninfected patients (mean age 46.7 (19.6) years).

Multivariate ANOVA revealed that two principal factors were independently associated with GMSH at all biopsy sites: $H$ pylori status $(p<0.0001)$ and age $(p<0.0001)$. Figure 1 shows the mean GMSH values of the whole sample measured at the three biopsy sites and subgrouped by $H$ pylori status. $H$ pylori infection was associated with a significantly decreased GMSH at the three gastric sites. There was no difference in GMSH between male and female subjects in any group (data not given). Small but significant differences were found between the biopsy sites, the contact angles in the antrum being significantly higher than in the corpus or cardia, both in patients with and without $H$ pylori infection.

Significant differences are as follows. At all sites: $H$ pylori negative greater than $H$ pylori positive (all $\mathrm{p}<0.0001)$. Infected patients: antrum greater than corpus $(\mathrm{p}=0.0001)$, antrum greater than cardia $(p=0.0362)$, cardia greater than corpus $(p=0.0041)$. Uninfected patients: antrum greater than corpus $(p=0.0003)$, antrum greater than cardia $(\mathrm{p}=0.0017)$.

To evaluate the age dependency of GMSH, three groups of equal size $(n=40)$ were formed to reflect the age distribution in the entire study population: group I (41 years or below); II (41-64 years); and III (65 years or above). On account of the well known age cohort effect the percentage of $H$ pylori infected patients increased through the groups, from $22.5 \%$ to $42.5 \%$ to $65 \%$, respectively. Figure 2 shows the mean GMSH contact angles measured at the three biopsy sites subgrouped by age (groups I-III) for patients without (fig 2A) and those with $H$ pylori infection (fig $2 \mathrm{~B}$ ). On comparison of $H$ pylori patients in age groups I and II, a significant decrease in GMSH was seen in the antrum only, while the decrease noted in 
Table 1 Means of contact angles in age groups I and III at the three gastric biopsy sites

\begin{tabular}{llll}
\hline & $\begin{array}{l}\text { Group I }(n=40) \\
\text { H pylori positive } \\
(n=9,22.5 \%)\end{array}$ & $\begin{array}{l}\text { Group III }(n=40) \\
\text { H pylori positive } \\
(n=26,65 \%)\end{array}$ & p Value \\
\hline $\begin{array}{l}\text { Antrum } \\
\text { H pylori negative }\end{array}$ & $71.0(4.8,69.2-72.8)$ & $65.5(3.4,63.5-67.4)$ & $<0.0001$ \\
$\begin{array}{c}\text { H pylori positive } \\
\text { Corpus }\end{array}$ & $66.6(2.5,64.6-68.5)$ & $61.5(3.7,60.0-63.1)$ & 0.001 \\
$\begin{array}{l}\text { H pylori negative } \\
\text { H pylori positive }\end{array}$ & $69.3(4.9,67.5-71.1)$ & $64.9(4.1,62.6-67.3)$ & 0.005 \\
$\begin{array}{c}\text { Cardia } \\
\text { H pylori } \text { negative }\end{array}$ & $63.3(3.1,60.9-65.7)$ & $60.5(3.4,59.1-61.9)$ & 0.041 \\
H pylori positive & $69.6(5.3,67.6-71.6)$ & $63.4(2.3,62.1-64.8)$ & $<0.0001$ \\
\hline
\end{tabular}

Results are expressed as mean (SD, 95\% confidence interval).

corpus and cardiac mucosa almost reached the level of significance. In uninfected patients, however, no effect was shown. Comparison of age groups II and III, in contrast, showed a significant decrease in contact angles in antral, corpus, and cardiac mucosa only in the absence of $H$ pylori while only a minor decrease is seen in $H$ pylori patients. Comparison of the age groups I and III clearly showed the effect of aging both in patients with and without $H$ pylori gastritis (table 1): significant decreases in mean GMSH values were found in antral, corpus, and cardiac mucosa. Figure 4 also illustrates this finding and shows the age dependent decrease in GMSH values measured in antral biopsy specimens from our overall study population subgrouped by $H$ pylori status. As shown in fig 3 and by the SD values in fig 2 , there were a few outliers with exceptionally low contact angles, especially among younger uninfected subjects. As all patients had undergone an interview to exclude
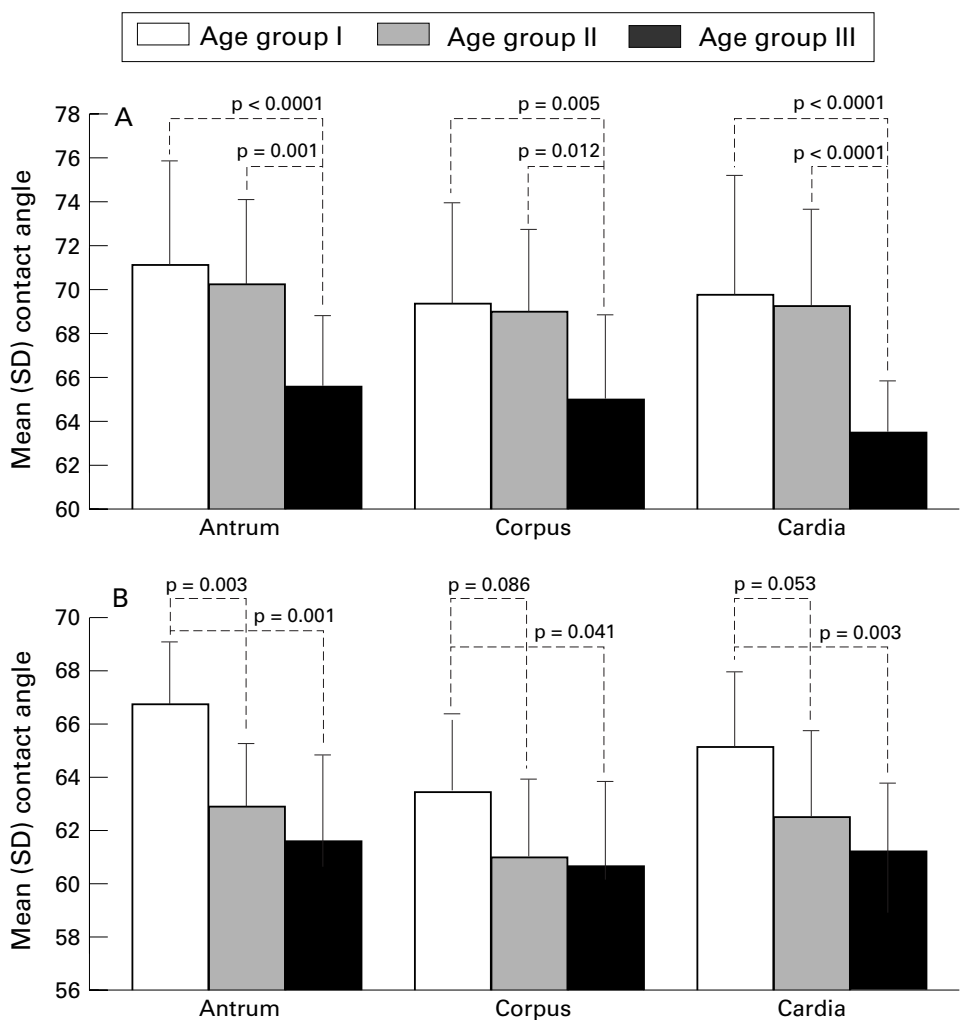

Figure 2 (A) Mean contact angles (SD) at the three biopsy sites in patients without $H$ pylori infection as a function of age. Group I $(n=31)$, Group II ( $n=23)$, Group III $(n=14)$. (B) Mean contact angles (SD) at the three biopsy sites in patients with H pylori infection as a function of age. Group I $(n=9)$, Group II $(n=17)$, Group III $(n=26)$.

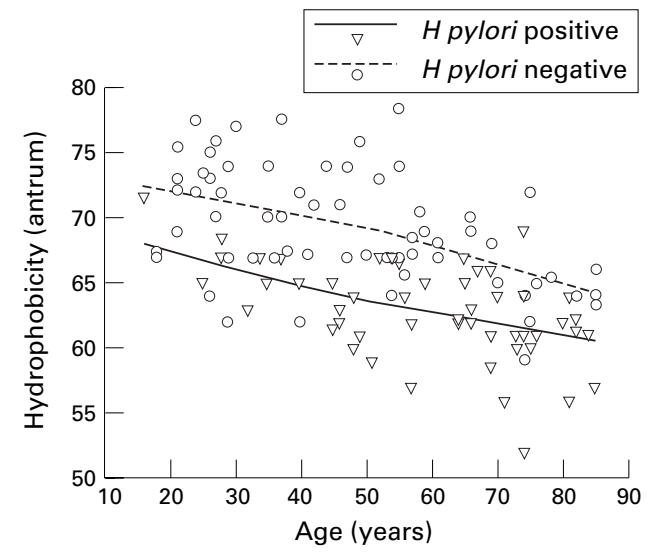

Figure 3 Contact angle values as a measure of GMSH in antral mucosa according to age and H pylori infection.

Table 2 Mean (SD) scores (Sydney System) for bacterial density, and active and chronic gastritis in $52 \mathrm{H}$ pylori infected patients

\begin{tabular}{llll}
\hline & Antrum & Corpus & Cardia \\
\hline H pylori density & $1.6(0.6)$ & $1.5(0.9)$ & $1.2(0.7)$ \\
Active gastritis & $1.5(0.6)$ & $1.4(0.7)$ & $1.3(0.5)$ \\
Chronic gastritis & $2.1(0.6)$ & $1.8(0.6)$ & $1.9(0.5)$ \\
\hline
\end{tabular}

Significant differences are as follows: $H$ pylori density: antrum $>$ cardia $(\mathrm{p}=0.001)$, corpus $>$ cardia $(\mathrm{p}=0.048)$; chronic gastritis: antrum $>$ corpus $(\mathrm{p}=0.002)$, antrum $>$ cardia $(\mathrm{p}=0.025)$.

the existence of factors known to influence hydrophobicity, we must ascribe this variation to unknown endogenous factors.

Histology in uninfected patients revealed no or only mild chronic inflammation (data not given). The mean scores of the $H$ pylori infected patients (table 2) revealed histological differences between the gastric sites with higher bacterial density and more notable chronic inflammation in antral compared with corpus or cardiac mucosa. Comparison of the histological scores in age groups I, II, and III revealed a slight, though insignificant $(\mathrm{p}<0.1)$, increase with age in bacterial density and active gastritis only in the corpus (detailed data not given). On evaluating all $52 \mathrm{H}$ pylori infected patients together, no significant association between GMSH and any of the histological variables was shown. On evaluating the age groups separately, however, group II showed a significant inverse relation between antral GMSH and the antral density of $H$ pylori $(r=-0.545, \mathrm{p}=0.024)$. No such correlations between GMSH and histology existed in corpus or cardiac mucosa. Analysis of the data revealed no outlying GMSH values associated with mucosal atrophy and/or intestinal metaplasia, neither of which was commonly seen in our sample (data not given). Of 52 infected patients, $30(57.7 \%)$ were seropositive for CagA and $21(40.4 \%)$ were seropositive for VacA by immunoblotting. However, neither marker of bacterial virulence showed any significant association with GMSH regardless of biopsy site (data not given).

\section{Discussion}

This study confirms earlier reports of reduced GMSH in $H$ pylori infected patients. ${ }^{10}{ }^{22}$ It has recently been reported ${ }^{23}$ that GMSH in antral 
biopsy specimens of infected patients was inversely related to the bacterial density in antral histology; in our sample this association was confirmed only in middle aged subjects. In addition we have established for the first time that in humans GMSH decreases with age independently of $H$ pylori status, the effect being seen equally in infected and uninfected individuals. Aging may therefore be added to $H$ pylori positive gastritis and NSAID medication as a factor impacting on gastric mucosal resistance. Our data show that this decrease in GMSH occurs at a younger age in $H$ pylori infected as compared with uninfected patients (figs 2 and 3). The most notable decrease in GMSH was seen between younger and middle aged $H$ pylori positive patients and apparently slowed above the age of 65 , while the histological severity of gastritis remained largely unchanged. In uninfected patients, however, the decrease was only seen in the elderly. It therefore seems possible that $H$ pylori infection aggravates and antedates a physiological impairment of mucosal resistance.

It has been shown in rabbits that GMSH is subject to maturation changes. In rabbit corpus mucosa the mean contact angle in suckling animals $\left(76.4^{\circ}\right)$ increases after weaning $\left(84.2^{\circ}\right)$ but decreases sharply in adult animals $\left(36.1^{\circ}\right) .{ }^{24}$ According to the commonly accepted model proposed by Lichtenberger et al, GMSH is determined by a mucous gel layer of adsorbed surface active phospholipids (SAPL). ${ }^{6}$ A phospholipid rich zone in the apical region of surface mucous cells has been shown ultrastructurally in endoscopic gastric biopsy specimens, ${ }^{25}$ and these phospholipids are a secretory component of gastric mucous cells. $^{7}$ A topical enhancement of hydrophobicity by oral SAPL, either in the form of a liposomal suspension or in milk has been shown in animal studies. ${ }^{6}{ }^{26-28}$ Thus an examination of GMSH in relation to dietary habits (consumption of dairy products) might be worthwhile. As all the patients in this study were investigated after an overnight fast, however, it is possible that acute nutritional influences could account for the variation of GMSH found in our sample.

Several studies on cultured gastric mucous cells have shown that prostaglandins $\left(\mathrm{PGE}_{2}\right)$ stimulate the synthesis of SAPL. ${ }^{29}{ }^{30}$ In the rat and canine gastric mucosa $\mathrm{PGE}_{2}$ has been shown to increase hydrophobicity and reverse the hydrophobicity reduced by aspirin. ${ }^{31-33}$

Several studies have investigated the effect of aging on gastric mucosal prostaglandin concentrations. An earlier study investigated the synthesis and catabolism of mucosal $\mathrm{PGE}_{2}$ in patients with gastritis and peptic ulcer, and found no clear correlation with age. ${ }^{34}$ More recently, two groups have reported a significant decrease in gastric mucosal prostaglandin content in the elderly. ${ }^{35}{ }^{36}$ One of these studies ${ }^{36}$ investigated asymptomatic patients with no history of PUD or NSAID usage and took the $H$ pylori status into account. Significantly decreased concentrations of $\mathrm{PGE}_{2}$ and $\mathrm{PGF}_{2 \alpha}$ were found in the elderly compared with younger patients. The mean $\mathrm{PGE}_{2}$ levels were higher in antral than in fundic mucosa, but this finding was not reported to be significant. Two studies investigating rat gastric mucosa found that mucosal prostaglandin synthesis decreased with age. ${ }^{37} 38$ A further mechanism relating to the age dependent decrease in GMSH might be the observation of a reduction in the number of epithelial cells in aged rats ${ }^{39}$ and of mucous cells in the gastric mucosa of elderly patients. ${ }^{40}$ However, these morphological changes may well be linked to a decrease in gastric mucosal prostaglandin concentrations in the elderly. ${ }^{41}$ In conclusion, the decreasing prostaglandin concentrations in the gastric mucosa of the elderly would seem to be a plausible explanation for the age dependent decrease in GMSH found in the present study.

This study shows that $H$ pylori gastritis weakens the mucosal defence in the cardia region, as also occurs in the corpus and antrum. ${ }^{42}$ In many infected patients, however, the bacterial density was higher and the mucosal inflammation more notable in the antrum than in corpus or cardia. This might explain in part, why in $H$ pylori positive patients the decline in GMSH with age was less pronounced in corpus than in antral mucosa. Furthermore, GMSH was higher in antral than in corpus mucosa in both $H$ pylori infected and uninfected patients. Such differences were not observed in the first study using endoscopic biopsy specimens of human gastric mucosa, ${ }^{9}$ but antral was found to be higher than corpus GMSH in both the pig $^{43}$ and the rabbit. ${ }^{24}$ Although our data cannot shed much light on these differences we suspect that they might in part be physiological. The differing amounts of mucus secreting versus specific (parietal and chief) cells in the antral and oxyntic mucosa ${ }^{44}$ might be a possible explanation.

In summary, aging reduces gastric mucosal surface hydrophobicity. This may act synergistically with the injurious effects of $H$ pylori gastritis and such exogenous compounds as NSAIDs on gastric mucosal defence and contribute to the higher risk for gastric ulcer development in the elderly. The hypothesis that gastric ulcer formation is more likely when gastric mucosal hydrophobicity falls short of a threshold level needs to be investigated further.

\footnotetext{
$1 \mathrm{NIH}$ Consensus conference. Helicobacter pylori in peptic ulcer disease. $\mathcal{F} A M A$ 1994;272:65-9.

2 Fries JF, Miller SR, Spitz PW, et al. Towards an epidemiology of gastropathy associated with nonsteroidal antiinflammatory drug use. Gastroenterology 1989;96:547-55.

3 Al-Assi MT, Genta RM, Karttunen TJ, et al. Ulcer site and Al-Assi MT, Genta RM, Karttunen TJ, et al. Ulcer site and
complications: relation to Helicobacter pylori infection and NSAID use. Endoscopy 1996;28:229-33.

4 El-Omar EM, Penman IA, Ardill JES, et al. Helicobacter pylori infection and abnormalities of acid secretion in patients with duodenal ulcer disease. Gastroenterology 1995;109:681-91.

5 Ruiz B, Correa P, Fontham E, et al. Antral atrophy, Helicobacter pylori colonization and gastric pH. Am $\mathcal{f}$ Clin Pathol 1996;105:96-101.

6 Lichtenberger LM, Graziani LA, Dial EJ, et al. Role of surface active phospholipids in gastric cytoprotection. Science 1983;219:1327-9.

7 Kao YC, Lichtenberger LM. Localization of phospholipidrich zones in rat gastric mucosa: possible origin of a protective hydrophobic luminal lining. I Histochem Cytochem 1987;35:1285-98.

8 Hills BA, Butler BD, Lichtenberger LM. Gastric mucosal barrier: the hydrophobic lining to the lumen of stomach. Am f Physiol 1983;244:G561-8.

9 Spychal RT, Marrero JM, Saverymuttu SH, et al. Measurement of surface hydrophobicity of human gastrointestinal mucosa. Gastroenterology 1989;97:104-11.
} 
10 Spychal RT, Goggin PM, Marrero JM, et al. Surface hydrophobicity of gastric mucosa in peptic ulcer diseaserelationship to gastritis and Campylo

11 Goddard PJ, Hills BA, Lichtenberger LM. Does aspirin damage canine gastric mucosa by reducing surface hydrophobicity? Am F Physiol 1987;252:G421-30.

12 Mendall MA, Goggin PM, Molineaux N, et al. Childhood living conditions and Helicobacter pylori seropositivity in adult life. Lancet 1992;339:896-7.

13 Cullen DJ, Collins BJ, Christiansen KJ, et al. When is Helicobacter pylori infection acquired? Gut 1993;34:16812.

14 Kurata JH. Ulcer epidemiology: an overview and proposed research framework. Gastroenterology 1989;96(suppl):56980.

15 Sipponen P, Seppälä K, Äärynen $M$, et al. Chronic gastritis and gastroduodenal ulcer: a case control study on the risk of coexisting duodenal or gastric ulcer in patients with gasof coexisting duodenal or gas
tritis. Gut 1989;30:922-9.

16 Lindell G, Celebioglu F, Stael von Holstein C, et al. On the natural history of peptic ulcer. Scand $\mathcal{F}$ Gastroenterol 1994 29:979-82.

17 Gabriel SE, Jaakkimainen L, Bombardier C. Risk for serious gastrointestinal complications related to use of nonsteroidal anti-inflammatory drugs. Ann Intern Med 1991;115 787-96.

18 Schubert TT, Bologna SD, Nensey Y, et al. Ulcer risk factors: interactions between Helicobacter pylori infection, nonsteroidal use and age. Am f Med 1993;94:413-18.

19 Dixon MF, Genta RM, Yardley JH, Correa P, and the participants in the international workshop on the histopathology of gastritis. Classification and grading of gastritis: the updated Sydney system. Am $\mathcal{F}$ Surg Pathol 1996;20:1161-81.

20 Malfertheiner P, Dominguez-Munoz JE, Heckenmüller $\mathrm{H}$, et al. Modified rapid urease test for detection of Helicobacter pylori infection. Eur $\mathcal{f}$ Gastroenterol Hepatol 1996;8:53-6.

21 Glupczynski Y, Vandenborre C, Goutier S, et al. Evaluation of Helicoblot 2.0-a new commercial Western blot of Helicoblot 2.0-a new commercial Western blot test - for the serological diagnosis of Helicobacte
infection [abstract]. Gut 1995;37(suppl 1):A59.

22 Goggin PM, Marrero JM, Spychal RT, et al. Surface hydrophobicity of gastric mucosa in Helicobacter pylori infection: effect of clearance and eradication. Gastroenterol ogy 1992;103:1486-90.

23 Asante M, Ahmed H, Patel P, et al. Gastric mucosal hydrophobicity in duodenal ulceration: role of Helicobacter pylori infection density and mucus lipids. Gastroenterology 1997;113:449-54.

24 Mack DR, Neumann AW, Policova Z, et al. Surface hydrophobicity properties of rabbit stomach in vitro. Pediatr Res 1994;35:209-13.

25 Mauch F, Bode G, Ditschuneit H, et al. Demonstration of a phospholipid rich zone in the human gastric epithelium damaged by Helicobacter pylori. Gastroenterology 1993; 105:1698-704.

26 Dial EJ, Lichtenberger LM. A role for milk phospholipids in protection against gastric acid. Studies in adult and suckling rats. Gastroenterology 1984;87:379-85.
27 Dial EJ, Lichtenberger LM. Milk protection against experimental ulcerogenesis in rats. Dig Dis Sci 1987;32:1145-50. 28 Kiviluoto T, Paimela H, Mustonen $\mathrm{H}$, et al. Exogenous surface active phospholipid protects Necturus gastric mucosa against luminal acid and barrier-breaking agents. Gastroenterology 1991;100:38-46.

29 Scheiman JM, Kraus ER, Bonnville LA, et al. Synthesis and prostaglandin $\mathrm{E}_{2}$ induced secretion of surfactant phospholipid by isolated gastric mucous cells. Gastroenterology 1991;100:1232-40.

30 Bernhard W, Schulte H, Piller M, et al. Synthesis and release of phosphatidylcholine by isolated porcine gastric mucous cells in primary culture. Eur f Clin Invest 1996;26:797-802.

31 Lichtenberger LM, Richards JE, Hills BA. Effect of 16,16-dimethyl prostaglandin $\mathrm{E}_{2}$ on the surface hydrophobicity of aspirin-treated canine gastric mucosa. Gastroenterology 1985;88:308-14

32 Dial EJ, Lichtenberger LM. Surface hydrophobicity in the developing rat. Effects of corticosteroids, thyroxine and prostaglandin $\mathrm{E}_{2}$. Gastroenterology 1988;94:57-61.

33 Goddard PJ, Kao YC, Lichtenberger LM. Luminal surface hydrophobicity of canine gastric mucosa is dependent on a surface mucous gel. Gastroenterology 1990;98:361-70.

34 Hawkey CJ. Synthesis of prostaglandin $\mathrm{E}_{2}$, thromboxane $\mathrm{B}_{2}$ and prostaglandin catabolism in gastritis and gastric ulcer. Gut 1986;27:1484-92.

35 Goto H, Sugiyama S, Ohara A, et al. Age-associated decreases in prostaglandin contents in human gastric mucosa. Biochem Biophys Res Commun 1992;186:1443-8.

36 Cryer B, Redfern JS, Goldschmiedt M, et al. Effect of aging on gastric and duodenal mucosal prostaglandin concentrations in humans. Gastroenterology 1992;102:118-23.

37 Uchida M, Kawano O, Misaki N, et al. Healing of acetic acid-induced gastric ulcer and gastric mucosal $\mathrm{PGI}_{2}$ level in rats. Dig Dis Sci 1990;35:80-5.

38 Lee $M$, Feldman M. Age-related reductions in gastric mucosal prostaglandin levels increase susceptibility to aspirin-induced injury in rats. Gastroenterology 1994;107: $1746-50$.

39 Majumdar AP, Jasti S, Hatfield JS, et al. Morphological and biochemical changes in gastric mucosa of aging rats. Dig Dis Sci 1990;35:1364-70.

40 Farinati F, Formentini S, Della- Libera G, et al. Changes in parietal and mucous cell mass in the gastric mucosa of normal subjects with age: a morphometric study. Gerontology 1993;39:146-51.

41 Majumdar AP, Fligiel SE, Jaszewski R. Gastric mucosal injury and repair: effect of aging. Histol Histopathol injury and repair:

42 Hackelsberger A, Günther T, Schultze V, et al. Prevalence and pattern of Helicobacter pylori gastritis in the gastric cardia. Am f Gastroenterol 1997;92:2220-4.

43 Goggin PM, Ahmed H, Northfield TC. Molecular basis for gastric mucosal hydrophobicity. Gastroenterology 1990; 98(suppl):A49.

44 Hogben AMC, Kent TH, Woodward PA, et al. Quantitative histology of the gastric mucosa: man, dog, cat, guinea pig and frog. Gastroenterology 1974;67:1143-54. 